\title{
A Foldable Antagonistic Actuator
}

\author{
Jun Shintake, Samuel Rosset, Bryan E. Schubert, Dario Floreano, Senior Member, IEEE, \\ and Herbert R. Shea, Senior Member, IEEE
}

\begin{abstract}
We report on an actuator based on dielectric elastomers that is capable of antagonistic actuation and passive folding. This actuator enables foldability in robots with simple structures. Unlike other antagonistic dielectric elastomer devices, our concept uses elastic hinges to allow the folding of the structure, which also provides an additional design parameter. To validate the actuator concept through a specific application test, a foldable elevon actuator with outline size of $70 \mathrm{~mm} \times 130 \mathrm{~mm}$ is developed with angular displacement range and torque specifications matched to a 400-mm wingspan micro-air vehicle (MAV) of mass $130 \mathrm{~g}$. A closed-form analytical model of the actuator is constructed, which was used to guide the actuator design. The actuator consists of $125-\mu \mathrm{m}$-thick silicone membranes as the dielectric elastomers, 0.2 mm-thick fiberglass plate as the frame structure, and 50 - $\mu \mathrm{m}$-thick polyimide as the elastic hinge. We measured voltage-controllable angular displacement up to $\pm 26^{\circ}$ and torque of $2720 \mathrm{mN} \cdot \mathrm{mm}$ at $5 \mathrm{kV}$, with good agreement between the model and the measured data. Two elevon actuators are integrated into the MAV, which was successfully flown, with the foldable actuators providing stable and well-controlled flight. The controllability was quantitatively evaluated by calculating the correlation between the control signal and the MAV motion, with a correlation in roll axis of over 0.7 measured during the flights, illustrating the high performance of this foldable actuator.
\end{abstract}

Index Terms-Antagonistic, artificial muscle, dielectric elastomer actuators (DEAs), foldable actuator, micro air vehicle.

\section{INTRODUCTION}

$\mathbf{F}$ OLDING technology in robotics has been explored as a means of self-assembly [1], programmable shape change [2], actuation [3], [4], structuring of printable robots, and micro robots [5]-[7], as well as the deployable wings of a jumpinggliding robot [8]. Moreover, folding technology improves portability by enabling efficient storage. In space engineering, the use of foldability has been investigated [9], and an example of such application can be seen in proposed aircraft for planetary exploration [10]. Foldability may be achieved with additional mechanisms which include mechanical parts like gears and linkages,

Manuscript received June 19, 2014; revised September 10, 2014; accepted September 16, 2014. Recommended by Technical Editor M. Moallem. This work was supported by the Swiss National Centre of Competence in Research Robotics and the Swiss National Science Foundation under Grant 200020140394.

J. Shintake is with the Microsystems for Space Technologies Laboratory, École Polytechnique Fédérale de Lausanne (EPFL), 2000 Neuchâtel Switzerland, and with the Laboratory of Intelligent Systems, EPFL, 1015 Lausanne, Switzerland (e-mail: jun.shintake@epfl.ch).

S. Rosset and H. R. Shea are with the Microsystems for Space Technologies Laboratory, École Polytechnique Fédérale de Lausanne, 2000 Neuchâtel, Switzerland (e-mail: samuel.rosset@epfl.ch; shea.herbert@epfl.ch).

B. E. Schubert and D. Floreano are with the Laboratory of Intelligent Systems, École Polytechnique Fédérale de Lausanne, 1015 Lausanne, Switzerland (e-mail: bryan.schubert@epfl.ch; dario.floreano@epfl.ch).

Color versions of one or more of the figures in this paper are available online at http://ieeexplore.ieee.org.

Digital Object Identifier 10.1109/TMECH.2014.2359337 and in some cases additional actuators dedicated to folding. This traditional mechanical approach may lead to increasing complexity of the structure, which results in increased design difficulty and fragility to external shocks and overload.

The use of soft actuators removes the need for complex mechanical assemblies and additional actuators, thus simplifying the structure by creating a compliant actuated joint that can passively fold. There are several types of existing soft actuator technologies [11]-[13], of which mainly three technologies are considered as candidates for this purpose: shape memory alloys (SMAs) [14], [15], pneumatic actuators [16], [17], and electroactive polymers (EAPs) [18].

SMAs exhibit high stress, self-sensing capabilities [19], and are able to provide compliance in spring configurations despite their high modulus of elasticity [20]. The response speed of SMAs is relatively slow, and there is hysteresis in an actuation cycle. SMAs can be driven with low voltage; however, they require high current and provide low efficiency. The thermoelastic behavior of SMA may also be affected by its external environment.

Pneumatic actuators can be formed with varied geometries and materials, and their actuation characteristics change from one type to another with the driving pressure. Therefore, it is difficult to compare them directly to SMAs and EAPs. However, pneumatic actuators exhibit high compliance when silicone elastomers are used, and they are able to provide high stress and large actuation strokes. Previously reported results show the existence of hysteresis in an actuation cycle [4], [21]. The need for an air compressor may result in bulky structures that prevent the mobility and the miniaturization of robots. Also, the materials used for pneumatic actuators generally do not have self-sensing capabilities.

EAPs can be divided into two major subcategories according to their actuation mechanism: ionic EAPs and electronic EAPs. The former type includes ionic polymer-metal composites (IPMCs) [22], [23], and the latter type holds dielectric elastomer actuators (DEAs) [24]-[27], both of which have been demonstrated for robotic devices. IPMCs are compliant, and able to provide large bending strokes with low actuation voltage. Also, they possess self-sensing capabilities. However, their response speed is slow (e.g., an IPMC actuator took $3.5 \mathrm{~min}$ to achieve $\sim 270^{\circ}$ of bending angle [28]), and their actuation characteristic exhibits a hysteresis. Moreover, stress and the efficiency are low. The slow motion of IPMCs is making them unsuitable to be applied to devices that require fast movements.

DEAs have high compliance ( $\sim 1 \mathrm{MPa}$ of modulus of elasticity), large actuation strokes (up to $85 \%$ of linear strain on a silicone elastomer [29]), fast response speed (kHz bandwidth [30]), theoretically high electromechanical efficiency (maximum 90\% [31]), and self-sensing capabilities [32]. In a silicone elastomer, 
the hysteresis in a cycle is relatively small due to low viscoelasticity [33]. Therefore, accurate positioning control is expected. In addition, a silicone elastomer also has a wide thermal tolerance, which supports the use of the actuator in external environments. However, DEAs usually generate low stresses, and a high voltage is necessary for driving them (typically a few $\mathrm{kV}$ ), which requires the use of dc/dc converters. Recent developments suggest potential solutions to these problems. Stacking DEAs can be used to improve the actuation stress [34]. Commercially available miniaturized dc/dc converters (e.g., EMCO Q series [35]) improve the mobility of robots and their miniaturization.

The aim of this study is to develop a foldable actuator based on a dielectric elastomer to enable foldability in robots with simple structures. The compliance of DEAs allows folding and gives robustness to external shocks and overload. Additionally, their highly elastic behavior allows for structures that self-deploy through the release of stored elastic strain energy.

In this paper, we first present the concept of a foldable actuator, and then validate the actuator through an application test. For the application, we develop a fixed wing microair vehicle (MAV) in which the actuator is used as an elevon. After the characterization of the elevon actuator, the actuator is evaluated in terms of the controllability of the MAV using the motion data obtained during flight. In Section II, we present the actuator and its characterization, and in Section III the MAV is developed. A discussion of the actuator is presented in Section IV followed by conclusion in Section $\mathrm{V}$.

\section{Foldable Antagonistic ACtUATOR}

\section{A. Actuator Concept}

DEAs consist of a thin elastomer membrane sandwiched between two compliant electrodes. When a voltage is applied onto the electrodes, the opposite charges on each electrode generate an electrostatic force (Maxwell stress) which squeezes the membrane and produces actuation stretch. This reduces the thickness of the membrane and its area expands in the case of freeboundary conditions. Existing actuator configurations exploit this working principle in many different ways [25], [26].

The actuator concept has an antagonistic configuration with two sets of stacked (or single) DEAs that allow bidirectional actuation and passive folding. Fig. 1 represents the mechanism of the actuator, in which Fig. 1(a) shows the nonactuated state. The actuator consists of rigid parts formed with two arms connected via elastic hinges that are symmetrically placed across the spacer and the DEAs. The DEAs are attached at their end points to the arms, while their centers are connected to the spacer such that they form an antagonistic configuration across the horizontal plane. All the DEAs have the same prestretch. The stretch in direction 1 is constant regardless of the actuation, and it is higher than the stretch in direction 2. Due to the prestretch, each DEA has an internal stress that keeps the actuator in its rest state.

According to the literature [24], [29], [36], the higher prestretch in direction 1 is expected to produce a large actuation stretch laterally perpendicular to its axis. This method has been applied to a cylindrical DEA configuration [37], which has shown a large actuation stroke. While several mechanisms

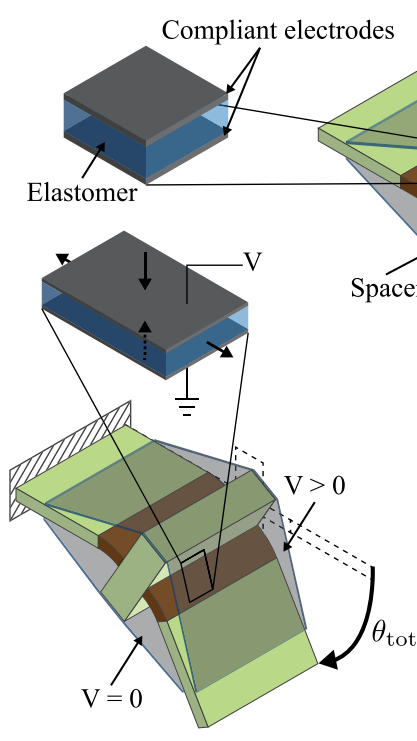

(b)

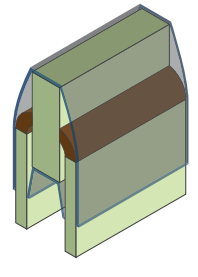

(c)
Fig. 1. Mechanism of the actuator. (a) Actuator consists of two rigid arms connected via elastic hinges placed symmetrically across the spacer, and two sets of stacked (or single) DEAs forming an antagonistic configuration. (b) When a voltage is applied to only the top or the bottom DEA, the electrostatic pressure causes a biased stress between the DEAs, resulting in a bending movement of the actuator. The stable position is determined by the angle at which the moment generated by the internal stresses in the DEAs and the counter moment in the hinges are equal. (c) Actuator can be passively folded where the internal stress of the DEA and the counter moment in the hinges generate a restoring force.

using an antagonistic configuration of DEAs have been developed [38], [39], our concept uses elastic hinges for supporting the folding of the structure and as an additional design parameter. The actuator output characteristics, such as the angular displacement and the torque, vary with the material properties or the geometry of the hinge. The characteristics can further be adjusted by changing the material properties or the geometry of the DEA. However, since many parameters are strongly related, it is often difficult to adapt a parameter in the DEA while keeping other parameters optimal (e.g., the thickness to maximize the electrostatic pressure or the prestretch to enlarge the actuation).

As shown in Fig. 1(b), when a voltage is applied to only the top or the bottom DEA of the actuator whose one tip is fixed, the electrostatic pressure in the DEA reduces the internal stress and causes a biased stress between the DEAs. The biased stress leads to a bending movement of the actuator. The stable position is determined by the angle at which the moment generated by the internal stresses in the DEAs and the counter moment in the hinges are equal. The amount of angular displacement depends on the voltage, the material properties, and the geometry of both the DEAs and the hinges. When the required performance cannot be achieved with a single DEA with fixed geometry, stacking of multiple DEA layers can be used to increase the actuator performance. In Fig. 1(b), the total angular displacement at the free tip $\theta_{\text {tot }}$ is twice the displacement obtained around a single hinge. The force produced at the free tip is determined only by the torque generated in the hinge closest to the fixed boundary, 


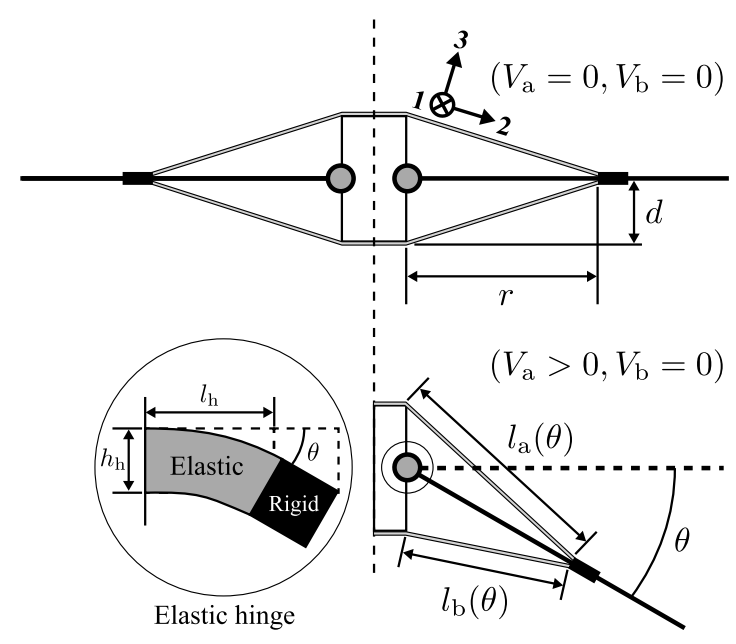

Fig. 2. Actuator model schematic. Due to symmetric structure, only half of the actuator is used for the model, while the elastic hinge is considered as a pivot.

that is the hinge closest to the free tip does not contribute to the force produced. Therefore, the output torque equals the moment of a single hinge with respect to its angular displacement. Due to the antagonistic configuration, the actuation can be done not only by a single voltage input but also by a combination of voltages on the DEAs on both sides. This suggests that variable mechanical impedance can be achieved where an angular displacement is provided with different output force.

As shown in Fig. 1(c), the actuator can be passively folded via the hinges. The DEA on the upper side is stretched and its internal stress is used as a restoring force towards the flat state. The counter moment in the hinges also contributes to the restoring force.

\section{B. Actuator Model}

A closed-form analytical model to predict the behavior of the actuator has been constructed based on the total potential energy in the system. The model outputs the total angular displacement $\theta_{\text {tot }}$ at an equilibrium state for a constant applied voltage $V_{\mathrm{s}}$. From this, the torque is obtained using the torsional spring constant of the elastic hinge. The subscript of variable, $s$, stands for either side of the actuator $(s=a$ or $b)$. The subscripts $a$ and $b$ stand for the upper side and the lower side of the actuator across the horizontal line, respectively. As previously mentioned, actuation can be done by a combination of voltages on the DEAs on both sides. However, for the rest of this paper, we consider the case of a single voltage input $\left(V_{\mathrm{a}}>0\right.$ and $V_{\mathrm{b}}=0$, or $V_{\mathrm{a}}=0$ and $V_{\mathrm{b}}>0$ ). As shown in Fig. 2, we only consider half of the actuator for the model since the structure is symmetric. The angular displacement $\theta$ is determined by calculating the local minimum of the total potential energy $U_{\text {tot }}$

$$
\frac{\partial U_{\text {tot }}}{\partial \theta}=0 \quad \text { and } \quad \frac{\partial^{2} U_{\text {tot }}}{\partial \theta^{2}}>0 .
$$

$U_{\text {tot }}$ consists of the potential energy of the DEA $U_{\mathrm{DEA}_{-} \mathrm{s}}$ and the elastic energy of the hinge $U_{\text {hinge }}$. Two energies are involved in $U_{\mathrm{DEA}_{-} s}$ : the strain energy of the DEA $U_{\text {strain_s }}$, and the electrostatic potential energy $U_{\text {electric_s }}$.

$$
\begin{aligned}
U_{\text {tot }}= & U_{\text {strain } \_\mathrm{a}}+U_{\text {strain } \_ \text {b }}+U_{\text {electric } \_a} \\
& +U_{\text {electric } \_b}+U_{\text {hinge }}
\end{aligned}
$$

where $U_{\text {strain_s }}$ is a function of the stretch of the DEA elastomer, in which the material property and the geometry are involved. To calculate $U_{\text {strain_s }}$, we assume that the elastomer is incompressible [29], [36], [40]

$$
\lambda_{1} \lambda_{2} \lambda_{3}=\lambda_{1 \mathrm{~s}} \lambda_{2 \mathrm{~s}} \lambda_{3 \mathrm{~s}}=1
$$

where $\lambda_{1}, \lambda_{2}$, and $\lambda_{3}$ are the stretch ratios of the elastomer on the direction of 1,2 , and 3 , respectively. The stretch ratio is defined as the ratio between the length of a deformed line element and its initial length. For each DEA, directions 1 and 2 refer to the planar directions of the DEA and direction 3 is along the thickness direction. To achieve the constant stretch in direction 1 during the fabrication, an elastomer with initial length $l_{0}$, initial width $w_{0}$, and initial thickness $h_{0}$, is prestretched uniaxially along direction 1 before attaching to the actuator structure. In this prestretched state, the geometry of the DEA is expressed as

$$
l_{\mathrm{s}}^{\prime}=\frac{l_{0}}{\sqrt{\lambda_{1 \mathrm{p}}}}, \quad w_{\mathrm{s}}^{\prime}=w_{0} \lambda_{1 \mathrm{p}}, \quad h_{\mathrm{s}}^{\prime}=\frac{h_{0}}{\sqrt{\lambda_{1 \mathrm{p}}}}
$$

where $l_{\mathrm{s}}^{\prime}, w_{\mathrm{s}}^{\prime}$, and $h_{\mathrm{s}}^{\prime}$ are the length, the width, and the thickness of the DEA, respectively, prestretched in direction 1 with a ratio of $\lambda_{1 \mathrm{p}}$. Since the stretch ratio in direction 1 is constant regardless of the actuation

$$
\lambda_{1 \mathrm{~s}}=\lambda_{1 \mathrm{p}}=\text { constant. }
$$

The DEA is then attached to the actuator structure with a stretch along direction 2, resulting in the assembled state shown as Fig. 2. From the figure and (4), $\lambda_{2 \mathrm{~s}}$ is obtained as the total stretch applied in both the prestretched state and the assembled state along its direction, that is

$$
\begin{aligned}
& \lambda_{2 \mathrm{a}}(\theta)=\frac{l_{\mathrm{a}}(\theta)}{l_{\mathrm{s}}^{\prime}} \cdot \frac{l_{\mathrm{s}}^{\prime}}{l_{0}}=\frac{l_{\mathrm{a}}(\theta)}{l_{0}}=\frac{\sqrt{r^{2}+d^{2}+2 d r \sin \theta}}{l_{0}} \\
& \lambda_{2 \mathrm{~b}}(\theta)=\frac{l_{\mathrm{b}}(\theta)}{l_{\mathrm{s}}^{\prime}} \cdot \frac{l_{\mathrm{s}}^{\prime}}{l_{0}}=\frac{l_{\mathrm{b}}(\theta)}{l_{0}}=\frac{\sqrt{r^{2}+d^{2}-2 d r \sin \theta}}{l_{0}}
\end{aligned}
$$

where $l_{\mathrm{a}}(\theta)$ and $l_{\mathrm{b}}(\theta)$ are the length of the DEA elastomer on each side, $r$ is the arm length, and $d$ is the height of the spacer from the arm. The stretch ratio along the thickness direction $\lambda_{3 \mathrm{~s}}$ is obtained by substituting (5) and (6) into (3)

$$
\lambda_{3 \mathrm{~s}}(\theta)=\frac{1}{\lambda_{1 \mathrm{p}} \lambda_{2 \mathrm{~s}}(\theta)} .
$$

We use the Yeoh hyperelastic model [41] as a strain energy density function $W$ to incorporate the stretch ratios in $U_{\text {strain_s }}$

$$
W=\sum_{i=1}^{3} C_{i}\left(I_{1}-3\right)^{i}
$$


where $C_{i}$ are material constants and $I_{1}=\lambda_{1}^{2}+\lambda_{2}^{2}+\lambda_{3}^{2}$. By substituting (5), (6), and (7) into (8), we get $U_{\text {strain_s }}$ as

$$
\begin{aligned}
U_{\text {strain_s }}(\theta) & =N_{\mathrm{s}} \cdot \mathrm{Vol} \cdot W_{\mathrm{s}}(\theta) \\
& =N_{\mathrm{s}} \cdot \mathrm{Vol} \cdot \sum_{i=1}^{3} C_{i}\left(I_{1 \mathrm{~s}}(\theta)-3\right)^{i} \\
I_{1 \mathrm{~s}}(\theta) & =\lambda_{1 \mathrm{p}}^{2}+\left\{\lambda_{2 \mathrm{~s}}(\theta)\right\}^{2}+\left\{\frac{1}{\lambda_{1 \mathrm{p}} \lambda_{2 \mathrm{~s}}(\theta)}\right\}^{2} \\
\mathrm{Vol} & =l_{0} w_{0} h_{0}
\end{aligned}
$$

where Vol is the volume of each DEA. Since the DEA can be stacked, we take into account the number of DEAs on each actuator side as $N_{s}$. As the DEA can be considered as a capacitor, the electrostatic potential energy $U_{\text {electric_s }}$ is derived as

$$
\begin{aligned}
U_{\text {electric } \_ \text {s }} & =-N_{\mathrm{s}} \frac{1}{2} C_{\mathrm{s}} V_{\mathrm{s}}^{2} \\
& =-N_{\mathrm{s}} \frac{1}{2} \varepsilon_{0} \varepsilon_{\mathrm{r}} \frac{A_{\mathrm{e}_{\mathrm{s}} \mathrm{s}}}{h_{\mathrm{s}}} V_{\mathrm{s}}^{2}
\end{aligned}
$$

where $C_{\mathrm{S}}$ is the capacitance, $\varepsilon_{0}$ is the permittivity of free space, $\varepsilon_{\mathrm{r}}$ is the relative permittivity of the elastomer, $A_{\mathrm{e}_{-} \mathrm{s}}$ is the area of the electrode, and $h_{\mathrm{s}}$ is the thickness of the elastomer. $U_{\text {electric_s }}$ is negative because we take into account the voltage-controlled case [42]. As the actuator moves with $\theta, A_{\mathrm{e}_{\mathrm{s}}}$ and $h_{\mathrm{s}}$ change corresponding to the stretch ratios, that is

$$
\begin{gathered}
A_{\mathrm{e} \_\mathrm{s}_{\mathrm{s}}}(\theta)=l_{\mathrm{e}_{-} \mathrm{s}}(\theta) w_{\mathrm{e}}=l_{\mathrm{e} 0} \lambda_{2 \mathrm{~s}}(\theta) w_{\mathrm{e}} \\
h_{\mathrm{s}}(\theta)=h_{0} \lambda_{3 \mathrm{~s}}(\theta)=\frac{h_{0}}{\lambda_{1 \mathrm{p}} \lambda_{2 \mathrm{~s}}(\theta)}
\end{gathered}
$$

where $l_{\mathrm{e}_{-} \mathrm{s}}$ and $w_{\mathrm{e}}$ are the length of the electrode and its width, respectively, and $l_{\mathrm{e} 0}$ is the electrode initial length. By substituting (11) into (10), $U_{\text {electric_s }}$ is represented as a function of $\theta$

$$
\begin{aligned}
U_{\text {electric_s }}(\theta)= & -N_{\mathrm{s}} \frac{1}{2} \varepsilon_{0} \varepsilon_{\mathrm{r}} l_{\mathrm{e} 0} w_{\mathrm{e}} \lambda_{1 \mathrm{p}} \\
& \times\left\{\lambda_{2 \mathrm{~s}}(\theta)\right\}^{2} \frac{V_{\mathrm{s}}^{2}}{h_{0}} .
\end{aligned}
$$

The elastic energy of the hinge, $U_{\text {hinge }}$, is obtained using the torsional spring constant $k$ and $\theta$

$$
U_{\text {hinge }}(\theta)=\frac{1}{2} k \theta^{2}
$$

where $k$ can be derived based on the small-length flexural pivot approximation (a pseudo-rigid-body model) [43]

$$
k=\frac{E_{\mathrm{h}} I_{\mathrm{h}}}{l_{\mathrm{h}}}=\frac{E_{\mathrm{h}} w_{\mathrm{h}} h_{\mathrm{h}}^{3}}{12 l_{\mathrm{h}}}
$$

where $E_{\mathrm{h}}$ and $l_{\mathrm{h}}$ are the modulus of elasticity of the hinge and its length, respectively, and $I_{\mathrm{h}}$ is the second moment of area. $I_{\mathrm{h}}$ is calculated using the width of the hinge $w_{\mathrm{h}}$ and its thickness $h_{\mathrm{h}}$.

As the energies $U_{\text {strain_s }}, U_{\text {electric_s }}$, and $U_{\text {hinge }}$ are functions of $\theta$, (1) becomes a differentiation of $U_{\text {tot }}$ with respect to $\theta$, that is

$$
\begin{aligned}
\frac{d U_{\text {tot }}}{d \theta}= & \frac{d U_{\text {strain_a }}}{d \theta}+\frac{d U_{\text {strain_b }}}{d \theta}+\frac{d U_{\text {electric } \_a}}{d \theta} \\
& +\frac{d U_{\text {electric_b }}}{d \theta}+\frac{d U_{\text {hinge }}}{d \theta}=0 .
\end{aligned}
$$

By differentiating (9), (12), and (13) with respect to $\theta$, each term in (15) is derived as

$$
\begin{aligned}
\frac{d U_{\text {strain_a }}}{d \theta}= & 2 N_{\mathrm{a}} \frac{w_{0} h_{0}}{l_{0}}\left(1-\frac{1}{\lambda_{1 \mathrm{p}}^{2}\left\{\lambda_{2 \mathrm{a}}(\theta)\right\}^{4}}\right) \\
& \times\left\{C_{1}+2 C_{2}\left(I_{1 \mathrm{a}}-3\right)\right. \\
& \left.+3 C_{3}\left(I_{1 \mathrm{a}}-3\right)^{2}\right\} d r \cos \theta \\
\frac{d U_{\text {strain_b }}}{d \theta}= & -2 N_{\mathrm{b}} \frac{w_{0} h_{0}}{l_{0}}\left(1-\frac{1}{\lambda_{1 \mathrm{p}}^{2}\left\{\lambda_{2 \mathrm{~b}}(\theta)\right\}^{4}}\right) \\
& +\left\{C_{1}+2 C_{2}\left(I_{1 \mathrm{~b}}-3\right)\right. \\
\frac{d U_{\text {electric_a }}=}{d \theta} & -N_{\mathrm{a}} \frac{\varepsilon_{0} \varepsilon_{\mathrm{r}} l_{\mathrm{e} 0} w_{\mathrm{e}}}{l_{0}^{2} h_{0}} \lambda_{1 \mathrm{p}} V_{\mathrm{a}}^{2} d r \cos \theta \\
\frac{d U_{\text {electric_b }}=}{d \theta} & N_{\mathrm{b}} \frac{\varepsilon_{0} \varepsilon_{\mathrm{r}} l_{\mathrm{e} 0} w_{\mathrm{e}}}{l_{0}^{2} h_{0}} \lambda_{1 \mathrm{p}} V_{\mathrm{b}}^{2} d r \cos \theta \\
\frac{d U_{\text {hinge }}}{d \theta}= & \frac{E_{\mathrm{h}} w_{\mathrm{h}} h_{\mathrm{h}}^{3}}{12 l_{\mathrm{h}}} \theta^{2} .
\end{aligned}
$$

By solving (15), we find $\theta$ as a function of the applied voltage $V_{\mathrm{s}}$, that is, $\theta=\theta\left(V_{\mathrm{s}}\right)$. As described in the previous section, the total angular displacement of the actuator $\theta_{\text {tot }}$ is twice the displacement obtained around a single hinge; therefore, $\theta_{\text {tot }}\left(V_{\mathrm{s}}\right)=2 \theta\left(V_{\mathrm{s}}\right)$.

The torque $\tau$ equals the moment in a single hinge and is a function of $V_{\mathrm{S}}$ in the case where the actuator tip is free to move

$$
\tau\left(V_{\mathrm{S}}\right)=k \theta\left(V_{\mathrm{S}}\right) .
$$

When the tip is blocked with a fixed angular displacement $\phi$, $\tau$ is obtained as

$$
\tau\left(V_{\mathrm{s}}, \phi\right)=k\left\{\theta\left(V_{\mathrm{s}}\right)-\phi\right\} .
$$

In (22), we assume that $\tau$ can be approximated to the value in the case of the free tip.

\section{Actuator Fabrication}

The desired specifications of performance and geometry of the actuator are obtained from the design process of the MAV described in Section III. The actuator is used as an elevon in the MAV and consists of a control surface and a base fixed onto the MAV airframe, which correspond to the arm parts shown in Fig. 1(a). Here, we present the fabrication process. The materials used for the fabrication were selected due to their lightweight property to minimize effects of both gravity and accelerations on the actuator movement. First, a silicone elastomer (Nusil, CF19-2186) is chosen for the DEA membrane. The elastomer 


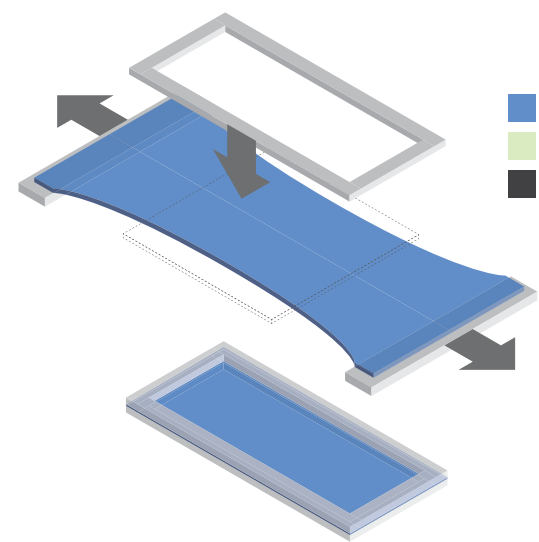

(a)

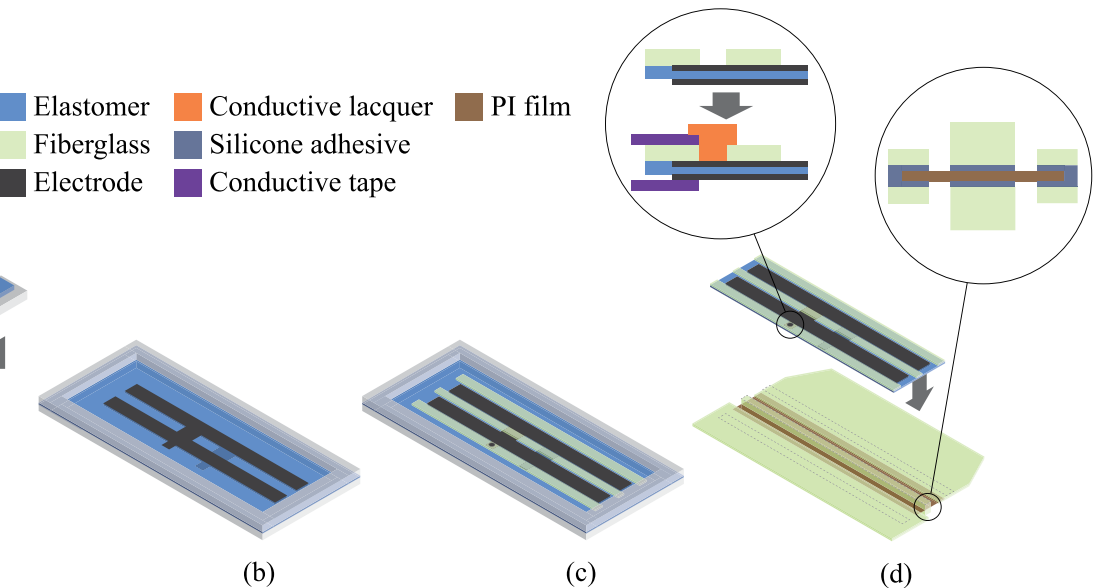

(c)

(d)

Fig. 3. Fabrication process of the actuator. (a) Elastomer is cut and peeled from a film substrate, prestretched uniaxially to a ratio of 2, and held by a supporting rack. (b) Electrodes are patterned on both side of the membrane via a stamping method. (c) Frames made of a fiberglass plate are attached on to the surface to keep the prestretched state. One of the frames has a hole for electrical contact. (d) Sample is then cut from the supporting rack and attached onto the actuator frame. The electrical contact is realized via a hole filled with a conductive lacquer and a conductive tape.

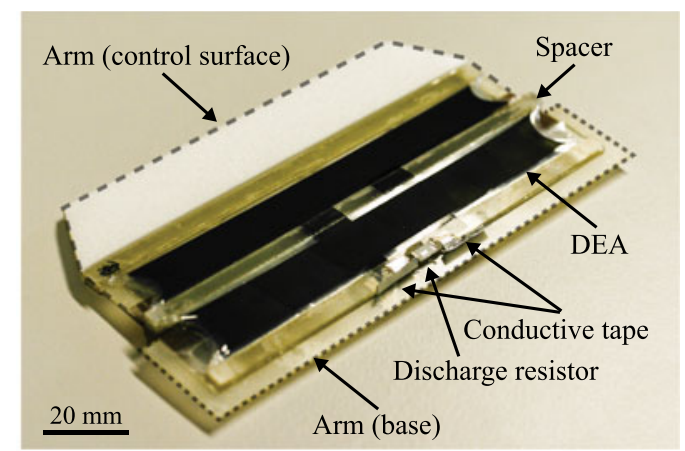

(a)

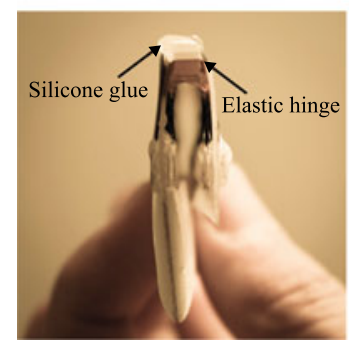

(b)

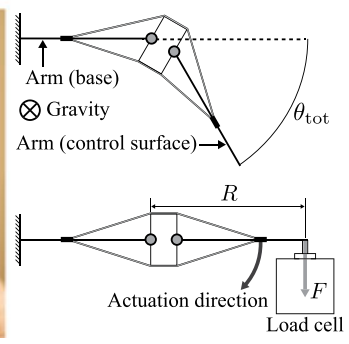

(c)
Fig. 4. (a) Elevon actuator. The actuator frame consists of two arm parts across the spacer: a control surface and a base. A resistor is connected between the conductive tape to discharge the DEA. (b) Folded state of the actuator. Silicone glue put on the frame edge prevents peeling of the DEA membrane. (c) Measurement condition of the actuator. For the total angular displacement $\theta_{\text {tot }}(=2 \theta$ in the model), the base tip is fixed and the displacement of the control surface tip is measured. For the torque, blocked force is measured. At a nonactuated state, the probe of the load cell is attached to the tip and subsequently the actuation voltage is applied. From the measured force, the torque is calculated using the moment arm length $R$ (in (22), $\phi=0$ ).

is mixed with a solvent (isooctane) at a 2:1 weight ratio, respectively, and the mixture is coated on a film substrate using an automatic film applicator coater (ZEHNTNER, ZAA2300) with a thickness given by a universal applicator (ZEHNTNER,
ZUA2000). After curing for $12 \mathrm{~h}$ at room temperature, an elastomer membrane with thickness of approximately $125 \mu \mathrm{m}$ is formed on the substrate.

The membrane is then cut into 80 -mm-long by 80 -mm-wide sections, peeled from the substrate, adhered to two rigid holders, and prestretched uniaxially to a ratio of 2 , as shown in Fig. 3(a). After that the sample is held by a supporting rack. The electrodes are subsequently patterned onto the membrane surface [see Fig. 3(b)] via a stamping method using a pad-printing machine (Teca-Print, TPM-101). For the electrode, we use a carbon-black-filled silicone. The geometry of the electrode is realized using a mask. The sample is cured for $1 \mathrm{~h}$ at $75^{\circ} \mathrm{C}$ after patterning one side, and the process is repeated on the other side of the membrane.

After curing, frames made of a 0.2-mm-thick fiberglass plate are attached to the membrane surface [see Fig. 3(c)] using a silicone adhesive film (Adhesives Research, ARclear-8932) to keep the prestretched state. One of the frame parts has a hole to make an electrical contact to the electrode. Additionally, a silicone glue is put on the edge of the frames to prevent the membrane from peeling. The sample is then cut from the supporting rack and attached to the actuator frame [see Fig. 3(d)]. The actuator frame is composed of two fiberglass plates of thickness 0.2 and $1 \mathrm{~mm}$, which are used as the arms (a control surface and a base) and the spacer, respectively, and a 50- $\mu$ m-thick polyimide (PI) film (UBE, UPILEX-50S) as the elastic hinges. The silicone adhesive film is used to attach them. The electrical contact is made via the hole of the frame using a conductive lacquer (amepox, ELECTON 40AC) and a conductive tape.

Fig. 4(a) shows the fabricated elevon actuator. The actuator consists of a control surface and a base as the arm parts. Fig. 4(b) shows the folded state of the actuator. The design parameters used for the actuator are summarized in Table I together with the specifications. In this table, the material constants $C_{i}$ used in (16) and (17) were determined by fitting (8) to the experimental stress-strain curve obtained from a uniaxial pull test (Noorwood, Instron 3343). 
TABLE I

DESIGN PARAMETER AND SPECIFICATIONS OF THE ELEVON ACTUATOR

\begin{tabular}{|c|c|}
\hline Design parameter & Value \\
\hline \multicolumn{2}{|l|}{ Dimensions } \\
\hline \multicolumn{2}{|l|}{ DEA (half part) } \\
\hline Initial length $l_{0}$ & $14.1 \mathrm{~mm}$ \\
\hline Initial width $w_{0}$ & $60 \mathrm{~mm}$ \\
\hline Initial thickness $h_{0}$ & $125 \mu \mathrm{m}$ \\
\hline \multicolumn{2}{|l|}{ DEA electrode (half part) } \\
\hline Initial length $l_{\mathrm{e} 0}$ & $11.3 \mathrm{~mm}$ \\
\hline Width $w_{\mathrm{e}}$ & $112 \mathrm{~mm}$ \\
\hline \multicolumn{2}{|l|}{ Frame } \\
\hline Arm length $r$ & $14.5 \mathrm{~mm}$ \\
\hline \multirow[t]{2}{*}{ Spacer height $d^{* 1}$} & $1.840 \mathrm{~mm}$ (one DEA) \\
\hline & $2.025 \mathrm{~mm}$ (two DEAs) \\
\hline Spacer area (length $\times$ width) & $4 \mathrm{~mm} \times 120 \mathrm{~mm}$ \\
\hline \multicolumn{2}{|l|}{ Elastic hinge } \\
\hline Length $l_{\mathrm{h}}$ & $1.0 \mathrm{~mm}$ \\
\hline Width $w_{\mathrm{h}}$ & $120 \mathrm{~mm}$ \\
\hline Thickness $h_{\mathrm{h}}$ & $50 \mu \mathrm{m}$ \\
\hline \multicolumn{2}{|l|}{ Silicone adhesive film } \\
\hline Thickness & $40 \mu \mathrm{m}$ \\
\hline \multicolumn{2}{|l|}{ Material property } \\
\hline \multicolumn{2}{|l|}{ DEA elastomer } \\
\hline Relative permittivity $\varepsilon_{\mathrm{r}}$ & $2.8[24],[30]$ \\
\hline Material constant $C_{1}$ & $0.105 \mathrm{MPa}$ \\
\hline Material constant $C_{2}$ & $0.00332 \mathrm{MPa}$ \\
\hline Material constant $C_{3}$ & $1.44 \times 10^{-13} \mathrm{MPa}$ \\
\hline \multicolumn{2}{|l|}{ Elastic hinge } \\
\hline Modulus of elasticity $E_{\mathrm{h}}^{* 2}$ & $9.1 \mathrm{GPa}$ \\
\hline \multicolumn{2}{|l|}{ Other parameter } \\
\hline Prestretch ratio $\lambda_{1 \mathrm{p}}$ & 2.0 \\
\hline Permittivity of free space $\varepsilon_{0}$ & $8.85 \times 10^{-12} \mathrm{~F} / \mathrm{m}$ \\
\hline \multicolumn{2}{|l|}{ Specifications } \\
\hline Mass & $14.4 \mathrm{~g}$ \\
\hline Base area (length $\times$ width) & $24 \mathrm{~mm} \times 130 \mathrm{~mm}$ \\
\hline Control surface area (length $\times$ width) & $40 \mathrm{~mm} \times 120 \mathrm{~mm}$ \\
\hline \multicolumn{2}{|c|}{$\begin{array}{l}{ }^{* 1} \text { Values are calculated as a composite of the fiberglass plate and } \\
\text { the silicone adhesive film. For the value of two DEAs, medium } \\
\text { height between both the DEAs was taken. } \\
{ }^{* 2} \text { According to the property of UPILEX-S presented on the man- } \\
\text { ufacturer's web site: http://www.ube.com/ }\end{array}$} \\
\hline
\end{tabular}

\section{Characterization}

1) Experimental Setup: We prepared two types of actuator samples: one consisted of one DEA on each side, and the other had two DEAs (in (16)-(19), $N_{\mathrm{s}}=1$ and $N_{\mathrm{s}}=2$ ). For each type, three samples were prepared and the average of the measured value was taken for the characterization. During the characterization, the total angular displacement $\theta_{\text {tot }}(=2 \theta$ in the model) and the blocked torque $\tau$ (in (22), $\phi=0$ ) were measured. The samples were fixed at the base with the direction minimizing gravity effects as shown in Fig. 4(c). The displacement of the control surface tip is measured as the angular displacement. A CMOS camera (Point Grey, FMVU-13S2C) with image processing was used to measure the angular displacement from the lateral side, and the torque was obtained by measuring the blocked force at the actuator tip using a load cell (FUTEK, LRF400). As shown in Fig. 4(c), the probe of the load cell was attached to the tip when the actuator was not actuated and after that the voltage was applied. The torque $\tau$ was then calculated from the measured force $F$ and the moment arm length $R$ as $\tau=R \cdot F$. Since the structure of the actuator is symmetric, only one side of the DEA was actuated with a voltage up to $5 \mathrm{kV}$ using a high-voltage dc supply (Auckland Biomimetics Lab, EAP controller).

2) Results: The measured angular displacements from the initial position, and the model predictions as functions of the applied voltage are shown in Fig. 5(a). The angle increases with the voltage and the maximum angular displacement is $16.9 \pm 1.5^{\circ}$ for the one DEA actuator, and $26.1 \pm 1.6^{\circ}$ for the two DEAs actuator. Fig. 5(b) shows the measured blocked torque and the model prediction as functions of the applied voltage. The torque also increases with the voltage and the maximum torque is 1480 $\pm 60 \mathrm{mN} \cdot \mathrm{mm}$ for the one DEA actuator, and $2720 \pm 260$ $\mathrm{mN} \cdot \mathrm{mm}$ for the two DEAs actuator. In these figures, as the model predicted, the actuator performance changes with the applied voltage nonlinearly, and the number of DEAs on each side affects the performance directly. The model prediction shows good agreement to the measured data. The error between the model prediction and measured data may have resulted from the fact that the model does not account for the Mullins effect [44], [45], the softening of the elastomer due to the prestretch, and the actuation stretch. The presence of the electrode elastomer, which is not accounted for in the model, would also decrease the actuation stretch. Furthermore, the actuator frame is not perfectly rigid so it may buckle when actuated, which could reduce the blocked force measured and, therefore, the torque. The error is larger in the case of the two DEAs actuator. This may have resulted from the fact that the geometry of each DEA is slightly different due to fabrication error, which would increase the error with number of DEAs attached to the actuator. Also, alignment error of those DEAs may raise the deviation between the model prediction and the measured data.

For the MAV, we used the actuator composed of two DEAs on each side as it exhibited torque of $2720 \mathrm{mN}$. mm with angular displacement of $26^{\circ}$, which satisfy the required torque of $1780 \mathrm{mN} \cdot \mathrm{mm}$ with angular displacement of $15^{\circ}$ obtained in the following Section III.

\section{VALIDATION}

We develop a remotely controlled fixed-wing MAV as an application to validate the actuator. The actuator is used as an elevon and evaluated in terms of the controllability of the MAV. For the evaluation, the correlation between the control signal and the angular velocity of the MAV motion is calculated.

\section{A. Airframe and Control System}

The design of the airframe includes the determination of the actuator specification from the wing geometry together with the required actuator torque. Fig. 6(a) shows the designed geometry of the airframe. We use a tailless shape for the airframe due to its simpler form. On the airframe, two actuators are used as elevons. The wing geometry is obtained and scaled down with its span from the Swift II (MS Composit), which is a successful commercial model. During a flight, the actuator has to sustain an elevon angle with a torque against the airflow where the aerodynamic force is acting on its surface. The required torque is calculated using the flight speed, the elevon surface, and 


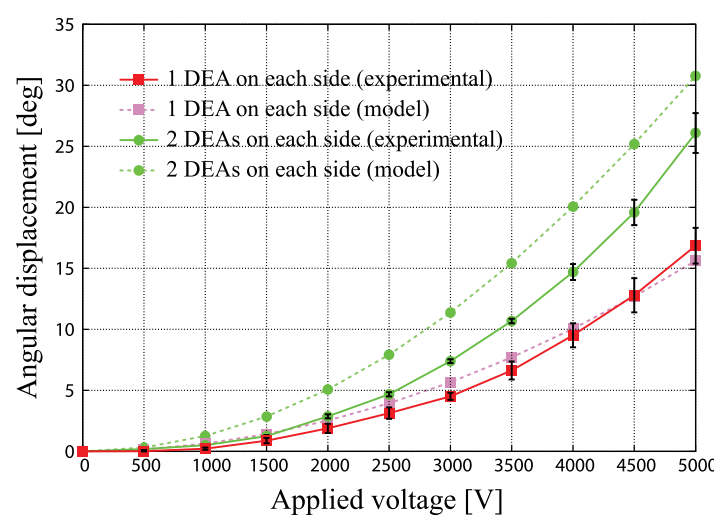

(a)

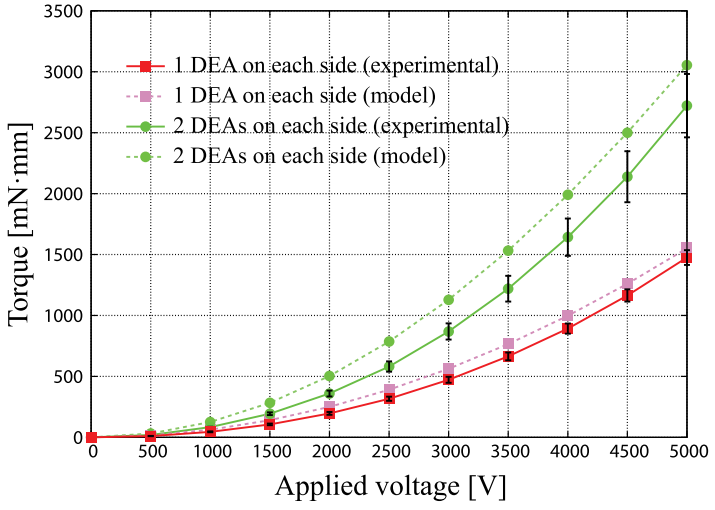

(b)

Fig. 5. Characterization results of two types of actuator samples: one consisted of one DEA on each side, and the other one had two DEAs. Only one side of the DEA was actuated for all the samples. (a) Measured angular displacement of one tip fixed condition and model prediction. The maximum angular displacement is $16.9 \pm 1.5^{\circ}$ for the one DEA actuator, and $26.1 \pm 1.6^{\circ}$ for the two DEAs actuator. (b) Measured blocked torque (in (22), $\left.\phi=0\right)$ and model prediction. The maximum torque was $1480 \pm 60 \mathrm{mN} \cdot \mathrm{mm}$ for the one DEA actuator, and $2720 \pm 260 \mathrm{mN} \cdot \mathrm{mm}$ for the two DEAs actuator.

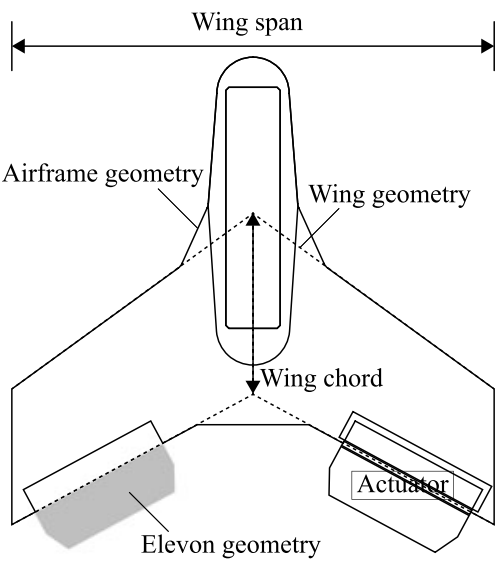

(a)

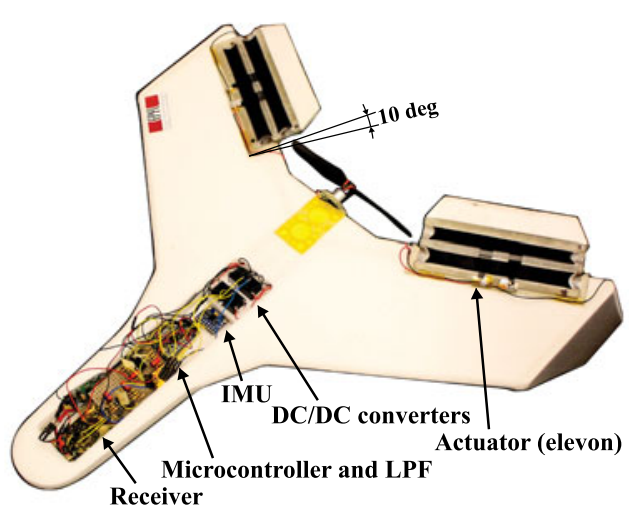

(b)

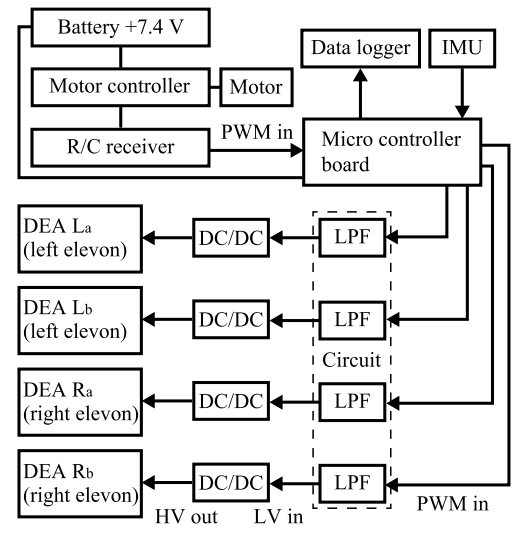

(c)

Fig. 6. (a) Schematic of the airframe. From a ratio of both the wing span and the chord, the elevon geometry is determined. Based on the geometry, the elevon actuator is realized. (b) Fabricated MAV (without the cover). The actuators are placed with mounting angle of $10^{\circ}$ to ensure the longitudinal static stability. (c) Control system of the MAV. The control signal from the receiver is converted to a high-voltage actuation signal for the DEAs via the microcontroller, the LPF circuit, and the dc/dc converters.

the elevon angle. During the flight, the mass of the MAV equals the lift force. The flight speed $V$ is determined as [46]

$$
V=\sqrt{\frac{2 m g}{C_{\mathrm{L}} \rho S}}
$$

where $C_{\mathrm{L}}$ is the lift coefficient, $\rho$ is the air density, $S$ is the wing area, $m$ is the mass, and $g$ is the gravity. $C_{\mathrm{L}}$ can be obtained as $C_{\mathrm{L}}=2 \pi \alpha$, and $\alpha$ is the angle of attack of the wing.

From (23), the required torque $\tau$ is obtained as

$$
\tau=r_{f} \cdot f=r_{f} \cdot \frac{1}{2} C_{\mathrm{Le}} \rho S_{\mathrm{e}} V^{2} \cos \beta
$$

where $f$ and $r_{f}$ are the aerodynamic force of the elevon and its position in the chord, respectively, $\beta$ the maximum elevon angle, $C_{\mathrm{Le}}$ is the lift coefficient of the elevon $\left(C_{\mathrm{Le}}=2 \pi \beta\right)$, and $S_{\mathrm{e}}$ is the elevon surface area. The elevon geometry $S_{\mathrm{e}}$ is obtained from a ratio of both the wing span and the chord shown in Fig. 6(a). $r_{f}$ can be approximated as $25 \%$ of the elevon chord, and we assume the aerodynamic force perpendicular to the elevon is a vector component of the elevon lift force. We use $15^{\circ}$ for $\beta$ because experimental results on thin wings at low Reynolds number have shown that the lift coefficient is linear within this region [47]. The elevon geometry is then used as the specification of the actuator. The nose of the airframe is extended to install the control system. Fig. 6(b) shows the fabricated MAV. We used 5-mm-thick styrofoam as an airframe material. The actuators are placed with a biased mounting angle of $10^{\circ}$ to ensure the longitudinal static stability as explained in Section III-B. The vertical tails are put downward to prevent damage to the actuators at landing. The specifications of the MAV are summarized in Table II.

The DEAs used for the actuator require high-voltage actuation signals; therefore, a dedicated control system is necessary for the MAV. Fig. 6(c) shows the outline of the control system. When a control signal is sent from the transmitter, the receiver (Futaba, R617FS) outputs a PWM signal, which is then 
TABLE II

SPECIFICATIONS OF THE MAV

\begin{tabular}{lc}
\hline \hline Specification & Value \\
\hline Dimensions & \\
$\quad$ Wing span & $400 \mathrm{~mm}$ \\
$\quad$ Wing chord & $150 \mathrm{~mm}$ \\
Wing area $S$ & $0.0525 \mathrm{~m}^{2}$ \\
Elevon surface area $S_{\mathrm{e}}$ & $0.0043 \mathrm{~m}^{2}$ \\
Elevon aerodynamic force position $r_{f}$ & $10.7 \mathrm{~mm}$ \\
Other & \\
$\quad$ Angle of attack of the wing $\alpha$ & $7.5^{\circ}$ \\
Maximum elevon angle $\beta$ & $15^{\circ}$ \\
Measured mass & $130.7 \mathrm{~g}$ \\
Estimated flight speed $V$ based on $m$ & $6.14 \mathrm{~m} / \mathrm{s}$ \\
$\quad$ Estimated required torque $\tau$ & $1780 \mathrm{mN} \cdot \mathrm{mm}$ \\
\hline \hline
\end{tabular}

processed via the micro-controller board (SparkFun, Arduino Pro Mini 328) to four outputs corresponding to DEAs placed in the actuators. Subsequently, the outputs are low-pass filtered to a low dc signal from PWM, and then the high-voltage signal is generated through the dc/dc converters (EMCO, Q-50, power $0.5 \mathrm{~W}$ ). Finally, a high-voltage output proportional to the low-voltage input is applied to the DEAs, up to a maximum of $5 \mathrm{kV}$. An IMU board (Kootek, GY-521) consisted of accelerometer and gyroscope is used to measure the motion of the MAV. During a flight, the sensor values are recorded together with the control signal by a data logger (SparkFun, DEV-09530). Since we analyze the data offline, the IMU in this system is not related to the control of the MAV.

From the point of view of the actuation, the resistor connected to the DEA dissipates power from the $\mathrm{dc} / \mathrm{dc}$ converter when the DEA is actuated, which is dominant power consumption. As shown in Fig. 6(c), there are four dc/dc converters in the MAV, and two of them are activated at same time for every maneuver. Thus, during a flight if the DEAs are actuated with maximum voltage all the time, the power consumption is approximated as $\sim 1 \mathrm{~W}$. We assume this value is acceptable for the MAV since the power consumption of the motor (HobbyKing, AP05 3000kv) is approximated as $\sim 22 \mathrm{~W}$ (calculated from the maximum current 3 A with driving voltage $7.4 \mathrm{~V}$ ), which is dominant in the entire system.

The control signal of the MAV consists of two channels: the pitch and the roll. As shown in Fig. 7(a), there are four DEAs as $L_{a}$ and $L_{b}$ for the left elevon, and $R_{a}$ and $R_{b}$ for the right elevon, respectively. Fig. 7(b) represents what appears when a pitch down is input. In this figure, the control signal and the actuation signal are normalized with respect to their maximum values, which are represented by the arrows. The signal is split out into two actuation signals and then $L_{a}$ and $R_{a}$ are actuated resulting in a down angle of both elevons which produces a nose-down pitch movement of the MAV during a flight. Table III summarizes the relation between the control signals and the corresponding actuated DEAs. For other cases, such as a combination of pitch and roll, actuation signal differences between $\mathrm{L}_{\mathrm{a}}$ and $\mathrm{L}_{\mathrm{b}}$, and between $\mathrm{R}_{\mathrm{a}}$ and $\mathrm{R}_{\mathrm{b}}$ are applied.

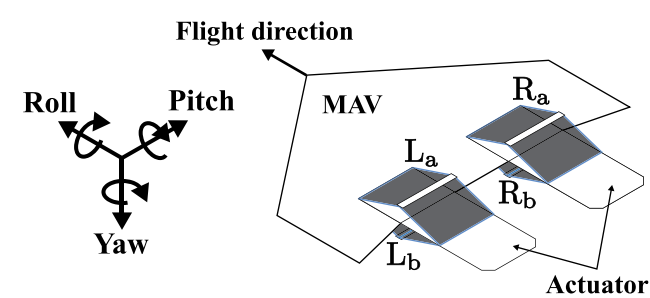

(a)

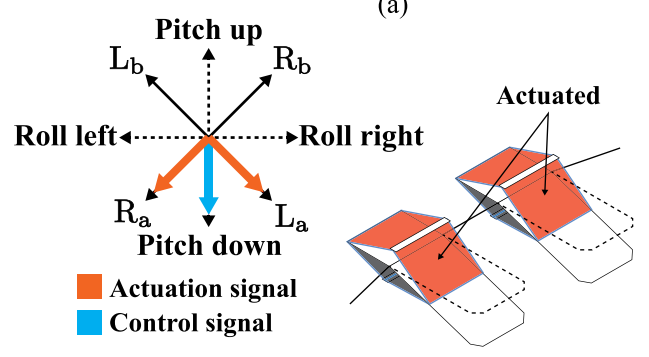

(b)

Fig. 7. Control and actuation signal relation. (a) There are four DEAs forming the elevons. $L_{a}$ and $L_{b}$ for the left elevon, $R_{a}$ and $R_{b}$ for the right elevon, respectively. (b) Control signal is split out to generate the actuation signal for those DEAs. In this case, $\mathrm{L}_{\mathrm{a}}$ and $\mathrm{R}_{\mathrm{a}}$ are actuated corresponding to a pitch down control signal.

TABLE III

CONTROL Signal versus ACTUATED DEA

\begin{tabular}{lcccc}
\hline \hline Control signal & DEA L $_{\mathrm{a}}$ & DEA L $_{\mathrm{b}}$ & DEA R $_{\mathrm{a}}$ & DEA R $_{\mathrm{b}}$ \\
\hline Pitch up & - & + & - & + \\
Pitch down & + & - & + & - \\
Roll left & - & + & + & - \\
Roll right & + & - & - & + \\
\hline \hline
\end{tabular}

+: actuated and -: not actuated.

\section{B. Results}

A flight of $150 \mathrm{~s}$ was performed, including an arm throw takeoff and a ground landing. During the flight, the MAV attitude control was achieved only by the elevon actuators via a remote controller with a human pilot. The data recorded during the flight has noise, which mostly comes from the motor. To suppress the noise, the data is smoothed with a low-pass filter (LPF) with a cutoff frequency of $1 \mathrm{~Hz}$. The filtered data range is a few seconds shorter than the flight time to avoid high noise coming from the takeoff acceleration and the landing deceleration. Also, the bias errors of the IMU are removed. The filtered data is then compared with the control signal, as shown in Fig. 8(a). The angular values refer to the coordinates shown in Fig. 7. The data on the yaw axis is not considered because it is a secondary factor based on the other two axes. The control signals are normalized with respect to their maximum value. The roll angular velocity visibly follows the control signal, while the pitch angular velocity shows low matching. The pitch angular velocity seems to be shifted positively. This is due to the fact that the MAV requires the pitch up angular velocity for turning, in addition to the roll and the yaw angular velocity. The correlation between the angular velocities and the control signals is 0.723 for the roll and 0.198 for the pitch. 


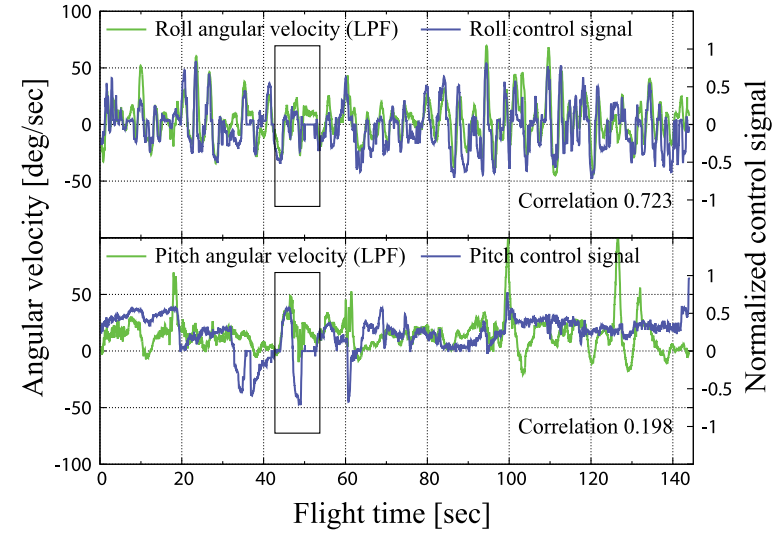

(a)

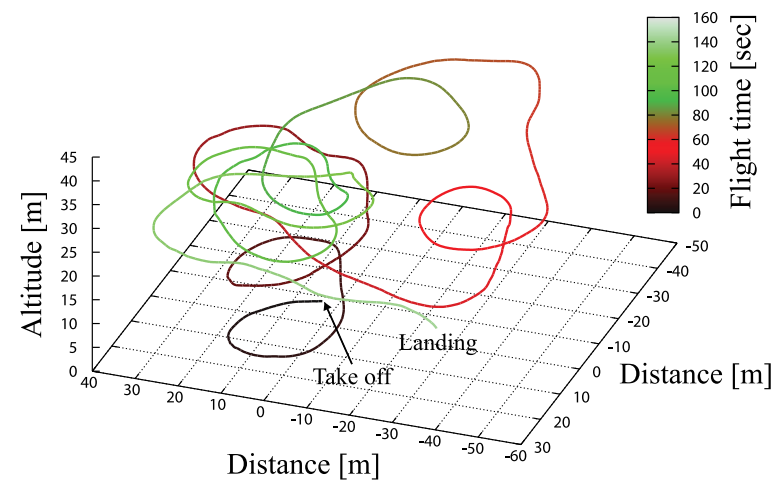

(b)

Fig. 8. (a) Comparison of the angular velocity and the control signal at flight time. During the flight, the attitude control was achieved only by the elevon actuators. The actuators controlled the MAV in the desired direction which is obvious from the fact that the angular velocity visibly follows the control signal. The trajectory shown in (b) is the result where the control signal therefore the will of the pilot is reflected. The trajectory is formed into a range where the MAV could be visually controlled.

The actuators controlled the MAV in the desired direction. This is obvious from the fact that the angular velocities, especially in the roll, visibly follow the control signal. This results in a strong correlation and, therefore, high controllability on this axis. Fig. 8(b) shows the flight trajectory of the MAV determined using the extended Kalman filter (EKF) [48]-[50] with the raw data of both the accelerometer and the gyroscope, while the flight speed is assumed to be constant, since the motor throttle was fixed during the flight except for the takeoff and the landing. The trajectory reflects the control signal (i.e., the will of the pilot) and forms a range where the MAV can be visually controlled.

The poor correlation with the pitch may have resulted from a strong longitudinal static stability of the MAV. The longitudinal static stability is necessary to keep the MAV attitude stable against disturbance (e.g., wind). Fig. 9(a) represents the MAV in steady flight. The static stability is achieved by the lift force and the aerodynamic force on the elevon actuators. Their moments around the center of gravity (COG) stabilize the MAV on the pitch axis. This inherent stabilization may resist controllability on this axis. Additionally, as shown in Fig. 9(b), due to the

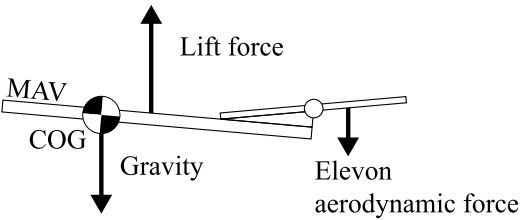

(a)

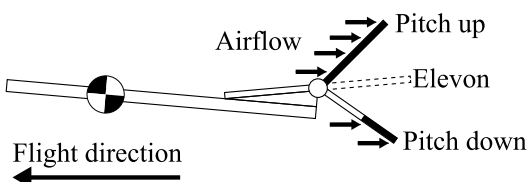

(b)

Fig. 9. (a) Longitudinal stability is achieved by the moments around the COG caused by the lift force and the aerodynamic force on the elevon, which are stabilizing the MAV attitude on the pitch axis. (b) Due to the biased actuator mounting angle, the surface area of the elevon exposed to the airflow is smaller at the direction of the pitch down, resulting in low controllability in this direction.

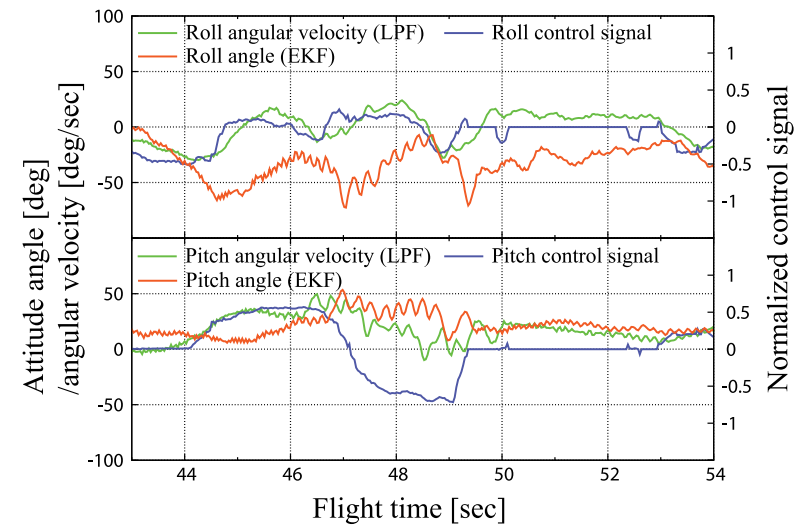

Fig. 10. Comparison of the attitude angle, the angular velocity, and the control signal at the flight time section of 43-54 s. The inherent stability is visible at $50-52 \mathrm{~s}$ where the attitude angles gradually decrease with no control signal towards their level flight attitude.

biased angle, the elevon surface exposed to the airflow is smaller at the pitch down; therefore, low controllability in this direction is expected. The swept wing of the MAV also stabilizes the attitude along the roll axis. Nevertheless, the correlation of this axis is strong, suggesting that the elevon actuator has adequate performance for aerodynamic control.

Fig. 10 shows the MAV attitude angle, the angular velocity, and the control signal at the flight time section of 43-54 s represented as a box in Fig. 8(a). The attitude angle is determined using the extended Kalman filter. The attitude angles follow the control signal with a delay which may have resulted from the inertia of the MAV. The inherent stability is visible at $50-52 \mathrm{~s}$ where the attitude angles gradually decrease with no control signal towards their level flight attitude: $0^{\circ}$ for the roll and an angle of attack for the pitch, respectively. Both the roll and the pitch angular velocity show biases at 50-52 s. The bias on the roll is due to the inherent stability on this axis. The bias on the pitch angular velocity suggests that the MAV is turning while the attitude approaches to the level flight. As we expected, 
the pitch angular velocity and, hence, the attitude is sensitive to the pitch up but not the pitch down.

\section{DISCUSSION}

An actuator capable of antagonistic actuation and passive folding has been proposed, and an elevon actuator for an MAV has been developed. During the characterization, the actuator showed controllable angular displacement and torque as functions of the applied voltage. The actuators controlled the MAV attitude in the desired direction during the flight from a takeoff to a ground landing, which resulted in strong correlation between the control signal and the MAV motion in the roll axis.

The flight of the MAV, which moves rapidly and requires accurate and fast control, proves that the positioning control and the response speed of the actuator perform adequately for this type of application. As the reason for this, mainly two actuator features are considered. First, the use of a silicone type elastomer as a DEA material, which has low viscoelasticity, contributes to both the positioning control and the response speed. Second, the simple actuator structure without additional mechanical parts prevents transmission loss. The simple structure also leads to ease of design.

In the model constructed in this study, the presence of many design parameters suggests the possibility of achieving a required performance with different combination of the parameters, allowing for flexibility of design. This is supported by the result shown in Fig. 5 where the actuator performance directly changed with the number of DEA on each side. This flexibility could potentially be translated to scalability with proper dimensioning.

The symmetric configuration of the actuator in the motion plane suggests modularity. Considered from the fact that many robotic systems existing today consist of multi-degree-offreedom systems, it would be possible to realize those motions while holding the actuator features, that is foldability and compliance. Also, as shown in the elevon actuator, an asymmetric planar geometry can be used for the arm part. This shows potential for the construction of complicated geometry forming systems where advanced robotic functionalities are realized, such as programmable shape change and reconfiguration.

The compliance of the actuator gives robustness not only to the system but also to external environments. This would provide, for example, safe robot-human interactions and handling of fragile objects. Also, the compliance can be used as elastic strain energy storage, which enables self-deploying of robot structures without the need of actuation.

\section{CONCLUSION}

In this study, we have developed a foldable antagonistic actuator based on a dielectric elastomer. The actuator possesses a simple structure, which may lead to the easy design of folding mechanisms. Additionally, the provided compliance could make devices robust to external shocks and overload. To validate the actuator through an application test, an elevon actuator was developed with the specifications obtained from the design of an MAV and an analytical actuator model was constructed, which guided the actuator design. The characterization results showed controllable angular displacement and torque as functions of applied voltage, while the model prediction showed good agreement to the measured data. The presence of many design parameters in the model potentially leads to design flexibility, which could be translated to scalability with proper dimension.

After the characterization, the actuator was assembled into the MAV to evaluate its controllability by calculating the correlation between the control signal and the MAV motion. During a flight, the actuators controlled the MAV attitude in the desired direction including a takeoff and a ground landing resulting in strong correlation between the control signal and the MAV motion in roll axis.

Future work is to develop fully foldable robotic systems using the presented actuator. It would be interesting to create a completely foldable version of the current MAV because it would show how the foldability of the entire airframe affects the robot's performance. Other work includes exploration of different applications, sensor implementations for intelligent systems, and investigation of control strategies. Model modifications such as improvement of prediction accuracy, implementation of dynamic characteristics, and adaptation to multi-degreeof-freedom systems with further characterization and validation are also important. However, the actuator we developed here and the initial results provide a good starting point for the creation of robots. In addition to foldability, these robots could possess advanced functionalities such as safe human-robot interaction, programmable shape change, and reconfiguration thanks to their simple structure and inherent compliance.

\section{ACKNOWLEDGMENT}

The authors would like to thank the members of both the LMTS and the LIS, and J. Paik for their assistance and helpful discussions.

\section{REFERENCES}

[1] S. M. Felton, M. T. Tolley, C. D. Onal, D. Rus, and R. J. Wood, "Robot self-assembly by folding: A printed inchworm robot," in Proc. IEEE Int. Conf. Robot. Autom., Karlsruhe, Germany, May 2013, pp. 277-282.

[2] E. Hawkes, B. An, N. M. Benbernou, H. Tanaka, S. Kim, E. D. Demaine, D. Rus, and R. J. Wood, "Programmable matter by folding," Proc. Nat. Acad. Sci. U.S.A, vol. 107, no. 28, pp. 12441-12445, 2010.

[3] H. Okuzaki, T. Saido, H. Suzuki, Y. Hara, and H. Yan, "A biomorphic origami actuator fabricated by folding a conducting paper," J. Phys. Conf. Series, vol. 127, no. 1, pp. 012001-1-012001-7, 2008.

[4] R. V. Martinez, C. R. Fish, X. Chen, and G. M. Whitesides, "Elastomeric origami: programmable paper-elastomer composites as pneumatic actuators," J. Adv. Funct. Mater., vol. 22, no. 7, pp. 1376-1384, 2012.

[5] C. D. Onal, R. J. Wood, and D. Rus, "An Origami-inspired approach to worm robots," IEEE/ASME Trans. Mechatron., vol. 18, no. 2, pp. 430-438, Apr. 2013.

[6] A. M. Hoover and R. S. Fearing, "Fast scale prototyping for folded millirobots," in Proc. IEEE Int. Conf. Robot. Autom., Pasadena, CA, USA, May 2008, pp. 1777-1778.

[7] P. S. Sreetharan, J. P. Whitney, M. D. Strauss, and R. J. Wood, "Monolithic fabrication of millimeter-scale machines," J. Micromech. Microeng, vol. 22, no. 5, pp. 055027-1-055027-6, 2012.

[8] M. Kovač, W. Hraiz, O. Fauria, J. C. Zufferey, and D. Floreano, "The EPFL jumpglider: A hybrid jumping and gliding robot with rigid or folding wings," in Proc. IEEE Int Conf. Robot. Biomimetics., Phuket, Thailand, Dec. 2011, pp. 1503-1508. 
[9] K. Miura, "Method of packaging and deployment of large membranes," in Proc. 31st Congr. Int. Astronaut. Fed., Tokyo, Japan, Dec. 1980, pp. 1-10.

[10] R. D. Braun, H. S. Wright, and D. A. Spencer, "The Mars airplane: A credible science platform," presented at the IEEE Aerosp. Conf., Big Sky, MT, USA, Mar. 2004.

[11] J. D. W. Madden, N. A. Vandesteeg, P. A. Anquetil, P. G. A. Madden, and A. Takshi, "Artificial muscle technology: Physical principles and naval prospects," IEEE J. Oceanic Eng., vol. 29, no. 3, pp. 706-728, Jul. 2004.

[12] D. Trivedi, C. D. Rahn, W. M. Kier, and I. D. Walker, "Soft robotics: Biological inspiration, state of the art, and future research," Appl. Bionics Biomech., vol. 5, no. 3, pp. 99-117, 2008.

[13] S. Bauer, S. B. Gogonea, I. Graz, M. Kaltenbrunner, C. Keplinger, and R. Schwödiauer, "25th anniversary article: A soft future: From robots and sensor skin to energy harvesters," Adv. Mater., vol. 26, no. 1, pp. 149-162, 2014.

[14] L. M. Schetky, "Shape-memory alloys," in Kirk-Othmer Encyclopedia of Chemical Technology. Hoboken, NJ, USA: Wiley, 1982.

[15] D. C. Lagoudas, Shape Memory Alloys. Berlin, Germany: Springer, 2008.

[16] F. Daerden and D. Lefeber, "Pneumatic artificial muscles: Actuators for robotics and automation," Eur. J. Mech. Environ. Eng., vol. 47, no. 1, pp. 11-21, 2002.

[17] F. Ilievski, A. D. Mazzeo, R. F. Shepherd, X. Chen, and G. M. Whitesides, "Soft robotics for chemists," Angew. Chem. Int. Ed., vol. 50, no. 8, pp. 1890-1895, 2011.

[18] Y. Bar-Cohen, Electroactive Polymer (EAP) Actuators as Artificial Muscles: Reality, Potential, and Challenges, 2nd ed. Bellingham, WA, USA: SPIE, 2004

[19] C. C. Lan and C. H. Fan, "An accurate self-sensing method for the control of shape memory alloy actuated flexures," Sens. Actuators A, Phys., vol. 163 , no. 1, pp. 323-332, 2010.

[20] S. Kim, E. Hawkes, K. Cho, M. Jolda, J. Foley, and R. J. Wood, "Micro artificial muscle ber using NiTi spring for soft robotics," in Proc. IEEE/RSJ Int. Conf. Intell. Robot. Syst., St. Louis, MO, USA, Oct. 2009, pp. 2228 2234.

[21] C. P. Chou and B. Hannaford, "Measurement and modeling of McKibben pneumatic artificial muscles," IEEE Trans. Robot. Autom., vol. 12, no. 1, pp. 90-102, Feb. 1996.

[22] M. Shahinpoor and K. J. Kim, "Ionic polymer-metal composites: I. Fundamentals," Smart Mater. Struct., vol. 10, no. 4, pp. 819-833, 2001.

[23] B. Bhandari, G.-Y. Lee, and S.-H. Ahn, "A review on IPMC material as actuators and sensors: Fabrications, characteristics and applications," Int. J. Precision Eng. Manuf., vol. 13, no. 1, pp. 141-163, 2012.

[24] R. Pelrine, R. Kornbluh, Q. Pei, and J. Joseph, "High-speed electrically actuated elastomers with strain greater than 100\%," Science, vol. 287, no. 5454, pp. 836-839, 2000.

[25] P. Brochu and Q. Pei, "Advances in dielectric elastomers for actuators and artificial muscles," Macromol. Rapid Commun., vol. 31, no. 1, pp. 10-36, 2010.

[26] I. A. Anderson, T. A. Gisby, T. G. McKay, B. M. O’Brien, and E. P. Calius, "Multi-functional dielectric elastomer artificial muscles for soft and smart machines," J. Appl. Phys., vol. 112, no. 4, pp. 041101-1-041101-20, 2012.

[27] S. Rosset and H. R. Shea, "Flexible and stretchable electrodes for dielectric elastomer actuators," J. Appl. Phys. A, vol. 110, no. 2, pp. 281-307, 2013.

[28] S. Nemat-Nasser and Y. Wu, "Comparative experimental study of ionic polymer-metal composites with different backbone ionomers and in various cation forms," J. Appl. Phys., vol. 93, no. 9, pp. 5255-5267, 2003.

[29] S. Akbari, S. Rosset, and H. R. Shea, "Improved electromechanical behavior in castable dielectric elastomer actuators," J. Appl. Phys. Lett., vol. 102, pp. 071906-1-071906-5, 2013.

[30] R. Kornbluh, R. Peirine, Q. Pei, S. Oh, and J. Joseph, "Ultrahigh strain response of field-actuated elastomeric polymers," in Proc. SPIE, San Diego, CA, USA, Mar. 2000, vol. 3987, pp. 51-64.

[31] F. Carpi, D. D. Rossi, R. Kornbluh, R. Pelrine, and P. Sommer-Larsen, Dielectric Elastomers as Electromechanical Transducers: Fundamentals, Materials, Devices, Models and Applications of an Emerging Electroactive Polymer Technology. Amsterdam, The Netherlands: Elsevier, 2008.

[32] T. A. Gisby, E. P. Calius, S. Xie, and I. A. Anderson, "An adaptive control method for dielectric elastomer devices," in Proc. SPIE, San Diego, USA, vol. 6927, pp. 69271C-1-69271C-8, Mar. 2008.

[33] S. Michel, X. Q. Zhang, M. Wissler, C. Löwe, and G. Kovacs, "A comparison between silicone and acrylic elastomers as dielectric materials in electroactive polymer actuators," Polym. Int., vol. 59, no. 3, pp. 391-399, 2010.
[34] G. Kovacs, L. Düring, S. Michel, and G. Terrasi, "Stacked dielectric elastomer actuator for tensile force transmission," Sens. Actuators A, Phys., vol. 155, no. 2, pp. 299-307, 2009.

[35] EMCO High Voltage Corp. EMCO proportional voltage products [Online]. Available: http://www.emcohighvoltage.com/proportional-powersupply.php

[36] M. Kollosche, J. Zhu, Z. Suo, and G. Kofod, "Complex interplay of nonlinear processes in dielectric elastomers," J. Phys. Rev. E, vol. 85, no. 5 , pp. 051801-1-051801-4, 2012.

[37] J. Huang, T. Lu, J. Zhu, D. R. Clarke, and Z. Suo, "Large, uni-directional actuation in dielectric elastomers achieved by fiber stiffening," J. Appl. Phys. Lett., vol. 100, pp. 211901-1-211901-4, 2012.

[38] P. Lochmatter and G. Kovacs, "Design and characterization of an active hinge segment based on soft dielectric EAPs," Sens. Actuators A: Phys. vol. 141, no. 2, pp. 577-587, 2008.

[39] P. Chouinard and J. Plante, "Bistable antagonistic dielectric elastomer actuators for binary robotics and mechatronics," IEEE/ASME Trans. Mechatron., vol. 17, no. 5, pp. 857-865, Jan. 2012.

[40] Z. Suo, "Theory of dielectric elastomers," Acta Mechanica Solida Sinica, vol. 23 , no. 6, pp. 549-578, 2010.

[41] O. H. Yeoh, "Some forms of the strain energy function for rubber," Rubber Chem. Tech., vol. 66, no. 5, pp. 754-771, 1993.

[42] G. Kofod, W. Wirges, M. Paajanen, and S. Bauer, "Energy minimization for self-organized structure formation and actuation," J. Appl. Phys. Lett., vol. 90, pp. 081916-1-081916-3, 2007.

[43] L. L. Howell, "Compliant mechanisms," in 21st Century Kinematics, London, U.K.: Springer-Verlag, 2013, pp. 189-216.

[44] L. Mullins, "Softening of rubber by deformation," Rubber Chem. Tech., vol. 42, no. 1, pp. 339-362, 1969.

[45] S. Rosset, L. Maffli, S. Houis, and H. R. Shea, "An instrument to obtain the correct biaxial hyperelastic parameters of silicones for accurate DEA modelling," in Proc. SPIE, San Diego, USA, vol. 9056, pp. 90560M-190560M-12, Mar. 2014.

[46] J. D. Anderson, Fundamentals of Aerodynamics, 5th ed. Singapore: McGraw-Hill, 2011.

[47] A. Pelletier and T. J. Mueller, "Low Reynolds number aerodynamics of low-aspect-ratio, thin/flat/cambered-plate wings," J. Aircraft, vol. 37, no. 5, pp. 825-832, 2000.

[48] G. Welch and G. Bishop, "An introduction to the Kalman filter," Dept Comput. Sci., Univ. North Carolina, Chapel Hill, North Carolina, 1995.

[49] A. Kim and M. Golnaraghit, "A quaternion-based orientation estimation algorithm using an inertial measurement unit," in Proc. Position Location Navigation Symp., Monterey, CA, USA, Apr. 2004, pp. 268-272.

[50] A. M. Sabatini, "Quaternion-based extended Kalman filter for determining orientation by inertial and magnetic sensing," IEEE Trans. Biomed. Eng., vol. 53, no. 7, pp. 1346-1356, Jul. 2006.

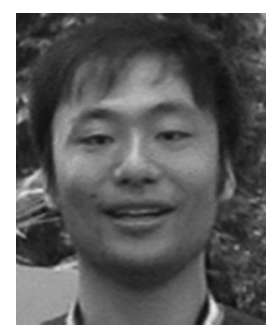

Jun Shintake received the B.Eng. M.Eng. degrees in mechanical engineering from the University of Electro-Communications, Tokyo, Japan, in 2009 and 2011, respectively. He is currently working toward the Ph.D. degree at the Laboratory of Intelligent Systems and the Microsystems for Space Technologies Laboratory, École Polytechnique Fédérale de Lausanne, Lausanne, Switzerland.

His research interests include smart materials, intelligent structure, and their use in the field of robotics especially soft, human-assist, bioinspired, reconfigurable, foldable, and aerial robotics.

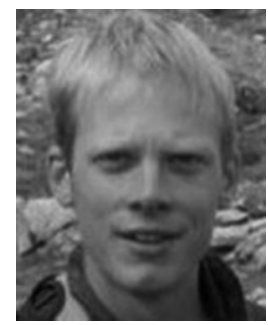

Samuel Rosset received the M.S. and Ph.D. degrees in microengineering from the École Polytechnique Fédérale de Lausanne (EPFL), Lausanne, Switzerland, in 2004 and 2009, respectively.

From 2005 to 2008, he was a Research Assistant with the microsystems for space technologies laboratory (LMTS) at EPFL. After a few years in industry developing tuneable optics based on soft elastomers, he reintegrated the LMTS in 2011 where he is now a Research Scientist, overseeing the activities on dielectric elastomer actuators. His research interests include soft actuators, compliant electrodes, development of new fabrication processes for soft sensors and actuators, and reliability and lifetime of these devices. 


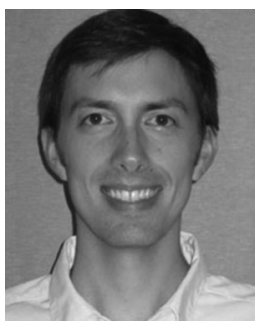

Bryan E. Schubert received the B.S. degree from Washington State University, Pullman, WA, USA, and the M.S. and Ph.D. degrees from the University of California, Berkeley, CA, USA, in 2005, 2007, and 2011, respectively, all in electrical engineering. He is currently a Postdoctoral Researcher at the Laboratory of Intelligent Systems at the École Polytechnique Fédérale de Lausanne, Lausanne, Switzerland. His research interests include the development of new actuators, sensors, and materials for soft robotics.

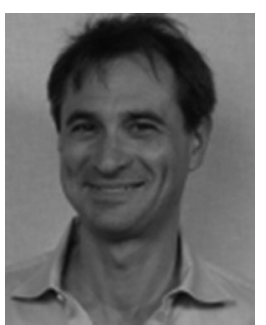

Dario Floreano (SM'06) received the M.A. and $\mathrm{Ph} . \mathrm{D}$. degrees from the University of Trieste, Trieste, Italy, in 1988 and 1995, respectively, and the M.S. degree from the University of Stirling, Stirling, Scotland, in 1991.

$\mathrm{He}$ is the Director of the Laboratory of Intelligent Systems, École Polytechnique Fédérale de Lausanne, Lausanne, Switzerland, and the Director of the Swiss National Center of Competence in Robotics. His research interests include the convergence of biology, artificial intelligence, and robotics. He published three books on the topics of evolutionary robotics, bioinspired artificial intelligence, and biomimetic flying robots with MIT Press and SpringerVerlag.

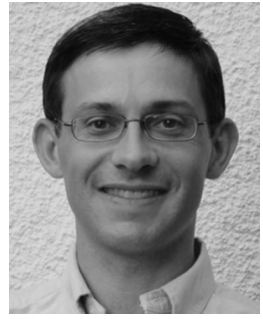

Herbert R. Shea (SM'09) received the B.Sc. degree from McGill University, Montreal, QC, Canada, and the Ph.D. degree from Harvard University, Cambridge, MA, USA, in 1991 and 1997, respectively, both in physics.

He developed carbon nanotube FETs at IBMs T. J. Watson Research Center from 1997 to 1999, then joined Lucent Technologies Bell Labs, Murray Hill, NJ, USA, where he became the Technical Manager of the Microsystems Technology group, specializing in MEMS reliability. In 2004 he founded the Microsystems for Space Technologies Laboratory, École Polytechnique Fédérale de Lausanne, Lausanne, Switzerland, where he is an Associate Professor, since 2011. His current research interests include micromachined soft polymer actuators and sensors, MEMS ion sources for micropropulsion of small spacecraft, and MEMS reliability. 